\title{
Title: $\mid$ Increasing the fracture toughness of silicon by ion implantation
}

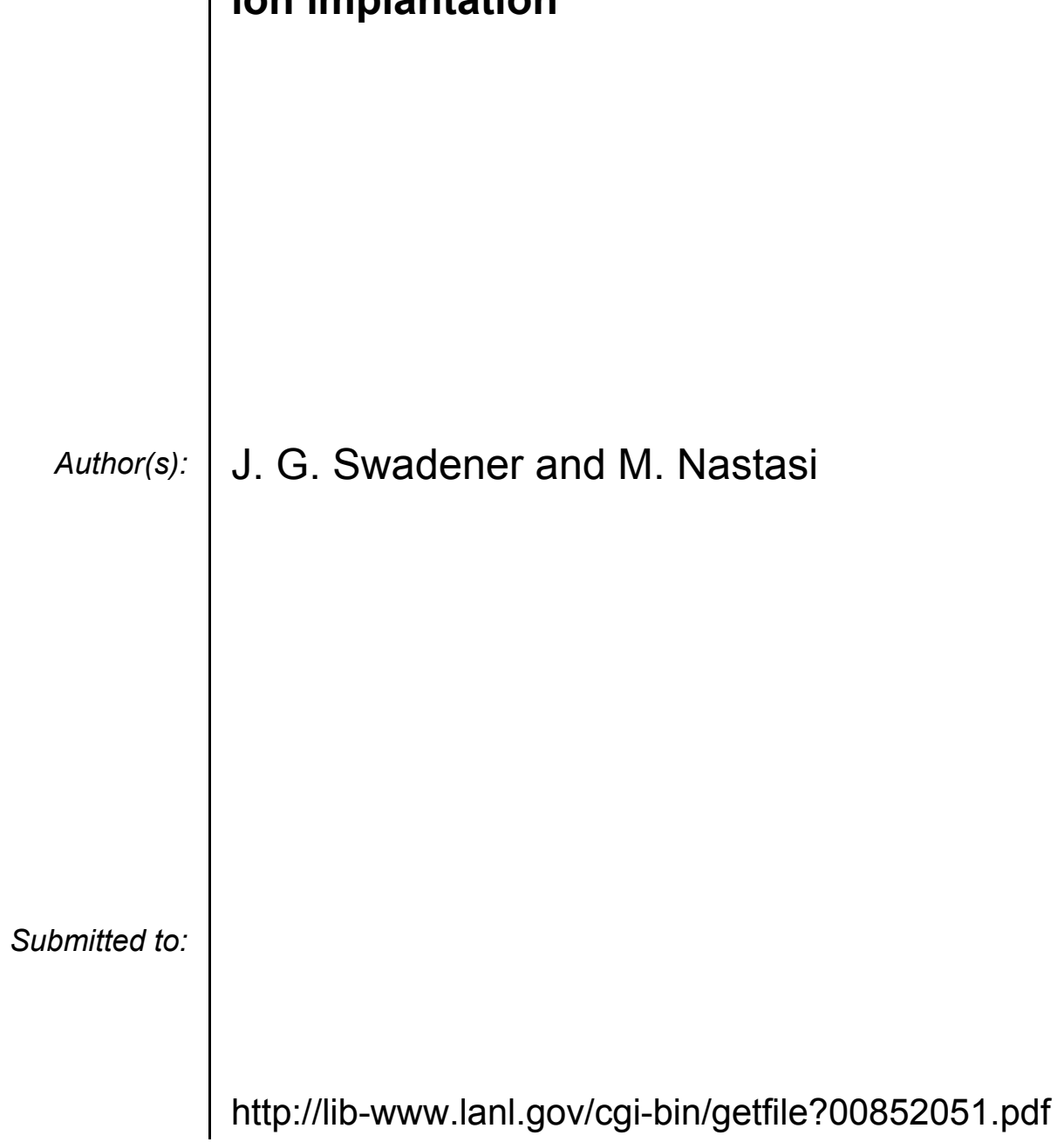

Los Alamos National Laboratory, an affirmative action/equal opportunity employer, is operated by the University of California for the U.S. Department of Energy under contract W-7405-ENG-36. By acceptance of this article, the publisher recognizes that the U.S. Government retains a nonexclusive, royaltyfree license to publish or reproduce the published form of this contribution, or to allow others to do so, for U.S. Government purposes. Los Alamos National Laboratory requests that the publisher identify this article as work performed under the auspices of the U.S. Department of Energy. Los Alamos National Laboratory strongly supports academic freedom and a researcher's right to publish; as an institution, however, the Laboratory does not endorse the viewpoint of a publication or guarantee its technical correctness. 


\title{
Increasing the fracture toughness of silicon by ion implantation
}

\section{J. G. Swadener and M. Nastasi, Materials Science \& Technology Div., Los Alamos National Laboratory, Los Alamos, NM 87545 USA}

Previous studies have shown that moderate doses of radiation can lead to increased fracture toughness in ceramics. An experimental investigation was conducted to determine the effects of ion implantation on fracture toughness in silicon. Specimens implanted with $\mathrm{Ne}$ showed increased fracture toughness, over the entire range of implantations tested. Using ions of various energies to better distribute implantation damage further increased the fracture toughness even though the region of amorphous damage was slightly decreased. The implantation damage accumulated in a predictable manner so that fracture toughness could be optimized.

PACS: $46.50+\mathrm{a}, 62.20 \mathrm{Mk}, 61.80 \mathrm{Jh}, 61.82 \mathrm{Fk}$

Keywords: fracture mechanics, implantation, radiation effects, silicon

\author{
J. G. Swadener \\ MST-8, MS-G755 \\ Los Alamos National Laboratory \\ Los Alamos, NM 87545 USA \\ e-mail: Swadener@lanl.gov \\ fax: 505-667-8021 \\ tel: 505-667-9952
}




\section{Introduction}

The effect of radiation on the fracture toughness of many materials has been investigated in numerous studies [e.g. 1-4]. The primary goal of most studies has been to determine the effect of a particular radiation environment on a candidate material. While the fracture toughness of most materials degrades with irradiation, an initial increase in fracture toughness (up to a $100 \%$ increase) has been observed experimentally in some ceramics, most noticeably in neutron irradiated Mg-Al spinel and alumina specimens [1]. Only a limited number of studies on of the effect of ion implantation have been conducted. Bolse et al. [2] found that implanting silicon ions in silicon nitride caused an initial modest increase in fracture toughness followed by a decrease in toughness at higher doses.

The current investigation was motivated by observations in hydrogen implanted silicon [5]. In that study, the location of microcracks in the implanted region was found to shift depending on the implanted dose. The implication is that implantation damage increased the fracture toughness of the implanted silicon, suppressing fracture in the center of the implanted region. This study experimentally measures the fracture toughness of $\mathrm{Ne}$ implanted silicon specimens in order to determine the relation between dose and fracture toughness. Furthermore, the effect of distributing implantation damage is investigated by using various ion energies that penetrate to different depths.

\section{Experimental Methods}

Specimens were cut from a single boron doped (100) silicon wafer, which had a resistivity less than $0.02 \Omega \mathrm{cm}$. Two series of implantations were conducted on the specimens. The first series used $300 \mathrm{keV}{ }^{20} \mathrm{Ne}^{++}$ions with doses of $5 \times 10^{14}, 1 \times 10^{15}, 2 \times 10^{15}, 5 \times 10^{15}$, and 
$2 \times 10^{16}$ ions $/ \mathrm{cm}^{2}$. For the second series, specimens were implanted with equal doses of 100,200 and $300 \mathrm{keV}{ }^{20} \mathrm{Ne}^{++}$ions. Doses of $5 \times 10^{14}, 1 \times 10^{15}, 2 \times 10^{15}$, and $5 \times 10^{15}$ ions $/ \mathrm{cm}^{2}$ for each ion energy were used in the second series. In order to minimize channeling effects, all implantations were conducted at an angle of $7^{\circ}$ from the surface normal. After implantation, the specimens were analyzed using Rutherford Backscattering Spectroscopy (RBS) in channeling mode. The detector was oriented at $13^{\circ}$ from the $2.0 \mathrm{MeV}^{4} \mathrm{He}^{+}$incident beam. Portions of the specimens were masked during implantation for comparison with the implanted regions.

Indentation fracture experiments were conducted on the implanted silicon specimens. Indentations were made to maximum loads of $1.0,1.5$ and $2.0 \mathrm{~N}$ using a Vickers tip. This load range typically produced radial cracks with radii $(c)$ greater than 3 times the indent with minimal collateral damage to the specimen. The indents were spaced a minimum of $10 c$ apart. The specimens were oriented so that fracture occurred primarily on (110) planes. A minimum of 20 tests were conducted for each specimen. Crack lengths were measured using an optical microscope and CCD camera, which gave a resolution of $0.46 \mu \mathrm{m}$.

The critical stress intensity factor $\left(K_{r}\right)$ was determined by using the formula derived by Lawn et al. [6]:

$$
K_{r}=\alpha\left(\frac{E}{H}\right)^{1 / 2} \frac{P}{c^{3 / 2}},
$$

where $\alpha$ is a constant, $E$ is the elastic modulus, $H$ is the hardness, and $P$ is the applied load. For a number of brittle materials, including silicon, Anstis et al. [7] found that $\alpha=0.016$ gave the best agreement with experimental results. This result has been verified by several investigations [e.g. 8, 9]. The fracture toughness $\left(G_{I C}\right)$ can be related to $K_{r}$ as: $G_{I C}=K_{r}^{2}\left(1-v^{2}\right) / E$, where $v$ is Poisson's ratio. 


\section{Results and Discussion}

In order to compare the damage produced by the two implantation series, the damage profiles produced from $\mathrm{Ne}$ implantation were calculated using SRIM [10]. The Si proton stopping coefficients that were used in the SRIM calculations were chosen to match experimental data. For $300 \mathrm{keV} \mathrm{Ne}$ ions, the predicted damage shows a peak at approximately 5000 Á, as shown in Fig. 1. For combined doses of 100, 200 and $300 \mathrm{keV} \mathrm{Ne}$ ions, the SRIM predictions show a damage peak at approximately $1800 \AA$, but overall, the damage is distributed more evenly than for the $300 \mathrm{keV}$ ions alone.

The damage produced in the implanted specimens was analyzed using RBS. The channeling data for $300 \mathrm{keV} \mathrm{Ne}$ implanted specimens are shown in Fig. 2. Damage is seen to increase monotonically with dose. For a dose of $5 \times 10^{15}$ ions $/ \mathrm{cm}^{2}$, a region of damage that appears to be amorphous is produced beginning at a depth of approximately $3000 \AA$ and extending to a depth of $7800 \AA$. For the maximum dose $\left(2 \times 10^{16}\right.$ ions $\left./ \mathrm{cm}^{2}\right)$, the damaged region extends from the surface to a depth of 7800 Á. Depths were calculated using RUMP simulations [11].

Figure 3 plots the channeling data for the second series of implants. For a dose of $1 \times 10^{15}$ ions $/ \mathrm{cm}^{2}$ at each ion energy, the damaged region is not yet amorphous, while twice that dose produces an apparently amorphous region extending from the surface to a depth of $7200 \AA$. Increasing the total dose to $1.5 \times 10^{16}$ ions $/ \mathrm{cm}^{2}$, extends the damaged region to a depth of $7700 \AA$.

Indentation fracture experiments were conducted on each implanted specimen and, for comparison, an unimplanted specimen. Eq. (1) predicts that, for constant material properties, the crack length to the $3 / 2$ power should increase linearly with the applied load. Plotting $P$ versus $c^{3 / 2}$ and using a least squares regression fit to the data provides a more accurate measure of $K_{r}$ than 
using a single load level. Additionally, the least squares curve fits to Eq. (1) gave a regression coefficient, $r$, greater that 0.98 for all specimens, which implies that the measured fracture toughness was not dependent on the crack length in the range tested $(12<c<30 \mu \mathrm{m})$.

The fracture toughness of the unimplanted specimen was determined to be $3.7 \mathrm{~J} / \mathrm{m}^{2}$, in agreement with previous results for (110) fracture in Si [7-9]. In Fig. 4, the fracture toughness results are plotted as a function of the integrated average damage predicted using SRIM. For specimens implanted only with $300 \mathrm{keV}$ ions, the fracture toughness increased with increasing damage over the entire range tested. However the fracture toughness increased only $5 \%$ when the dose increased from $5 \times 10^{15}$ ions $/ \mathrm{cm}^{2}$ to $2 \times 10^{16}$ ions $/ \mathrm{cm}^{2}$, while the size of the damage region increased $62 \%$.

The data suggests that if the implantation damage were distributed more evenly, fracture toughness could be further increased. As shown in Fig. 4, the fracture toughness results for specimens implanted with three different energies of ions demonstrates that distributing the damage increased fracture toughness compared to the specimens implanted with a single ion energy in all cases. Although the specimen implanted with a dose of $2 \times 10^{15}$ ions $/ \mathrm{cm}^{2}$ for each of the three ion energies had a smaller amorphous region and lower DPA than some specimens implanted with only $300 \mathrm{keV}$ ions, it had the maximum measured fracture toughness $\left(6.2 \mathrm{~J} / \mathrm{m}^{2}\right)$.

The indentation fracture method used here produced cracks that penetrated much deeper $(12-30 \mu \mathrm{m})$ than the implanted regions $(<1 \mu \mathrm{m})$. Therefore, the measured fracture toughness does not represent the toughness of the implanted region itself, but rather, gives a weighted average (weighted in favor of the surface region where crack lengths are measured) of the implanted region and the pristine silicon beneath it. The weighting factor has not yet been 
determined. The fracture toughness of the implanted region itself may be significantly higher than the measured values shown in Fig. 4.

\section{Conclusions}

Boron doped silicon specimens were implanted with Ne ions and the specimen fracture toughness was measured experimentally. Over the range of implantations tested, implanted specimens all showed an increase in fracture toughness compared to unimplanted silicon. Using ions of various energies to distribute the implantation damage further increased the specimen fracture toughness (compared to specimens implanted with a single energy) despite a reduction in the size and DPA of the implanted region.

\section{Acknowledgement}

This research was funded by the U. S. Department of Energy Office of Basic Energy Sciences.

Thanks to J. R. Tesmer, C. J. Wetteland and M. G. Hollander and R Schellenberg for helpful discussions and technical assistance. 


\section{References}

1. F. W. Clinard, Jr., G. F. Hurley, R. A. Youngman, and L. W. Hobbs, J. Nucl. Mater. 133\&134 (1985) 701 .

2. W. Bolse, S. D. Peteves and F. W. Saris, Appl. Phys. A 58 (1994) 493.

3. Y. Matsui, M. Niimi, T. Hashiy, F. Sakurai, S. Jitsukawa, T. Tsukada, M. Ohmi, H. Skai, R. Oyamada, and T. Onchi, J. Nucl. Mater. 233-237 (1996) 188.

4. T. Yano, J. Am. Ceram. Soc. 82 (1999) 3355.

5. T. Höchbauer, A. Misra, M. Nastasi, J. W. Mayer, J. Appl. Phys. 92 (2002) 2335.

6. B. R. Lawn, A. G. Evans and D. B. Marshall, J. Am. Ceram. Soc. 63 (1980) 574.

7. G. R. Anstis, P. Chantikul, D. B. Marshall, and B. R. Lawn, J. Am. Ceram. Soc. 64 (1981) 533.

8. B. R. Lawn, D. B. Marshall and P. Chantikul, J. Mater. Sci. 16 (1981) 1769.

9. M. Nastasi, P. Kadali, K. C. Walter, J. D. Embury, R. Raj and Y. Nakamura, J. Mater. Res. 14 (1999) 21739.

10. J. F. Ziegler, J. B. Biersack and U. Littmark, The Stopping and Range of Ions in Solids (Pergamon, New York, 1985).

11. M. Thompson and L. Doolittle, RUMP - RBS Analysis and Simulation Package, v.4.0 (2002). 
Figure Captions

Fig. 1. SRIM [11] predictions of damage from Ne ion implantation in $\mathrm{Si}: 100 \mathrm{keV}$ ions (dotted line), $200 \mathrm{keV}$ ions (dashed), $300 \mathrm{keV}$ ions (long dash) and sum of all three (solid line).

Fig. 2. RBS-channeling spectra of $\mathrm{Si}$ specimens implanted with $300 \mathrm{keV} \mathrm{Ne}$ ions (doses as indicated).

Fig. 3. RBS-channeling spectra of Si specimens implanted with equal doses (indicated) of 100, 200 and $300 \mathrm{keV} \mathrm{Ne}$ ions.

Fig. 4. Increase in fracture toughness (relative to unimplanted specimens) of Ne implanted $\mathrm{Si}$ specimens: $300 \mathrm{keV}$ ions (circles) and combined 100, 200 and $300 \mathrm{keV}$ ions (squares). 


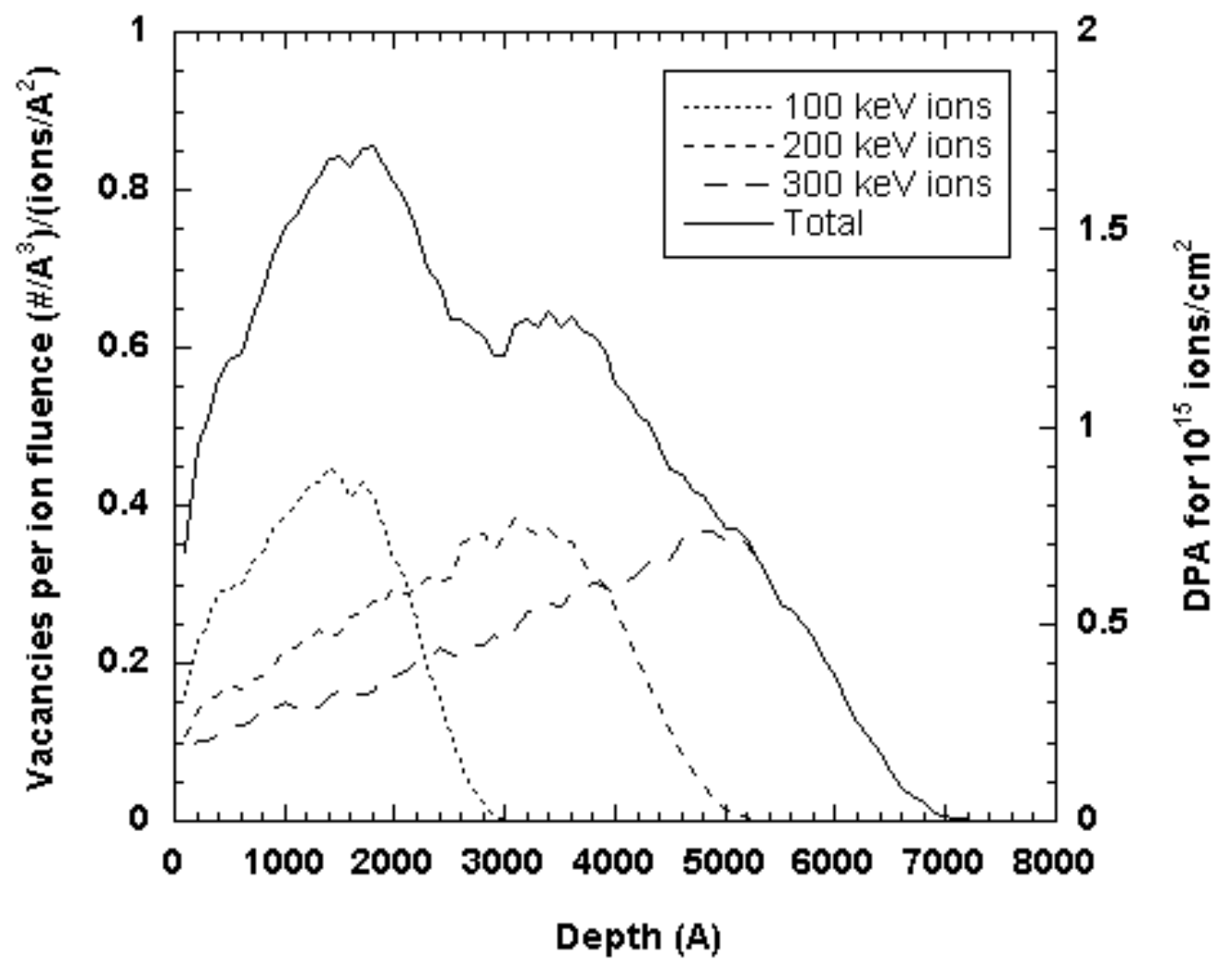

Fig. 1. SRIM [11] predictions of damage from Ne ion implantation in Si:100 keV ions (dotted line), $200 \mathrm{keV}$ ions (dashed), $300 \mathrm{keV}$ ions (long dash) and sum of all three (solid line). 


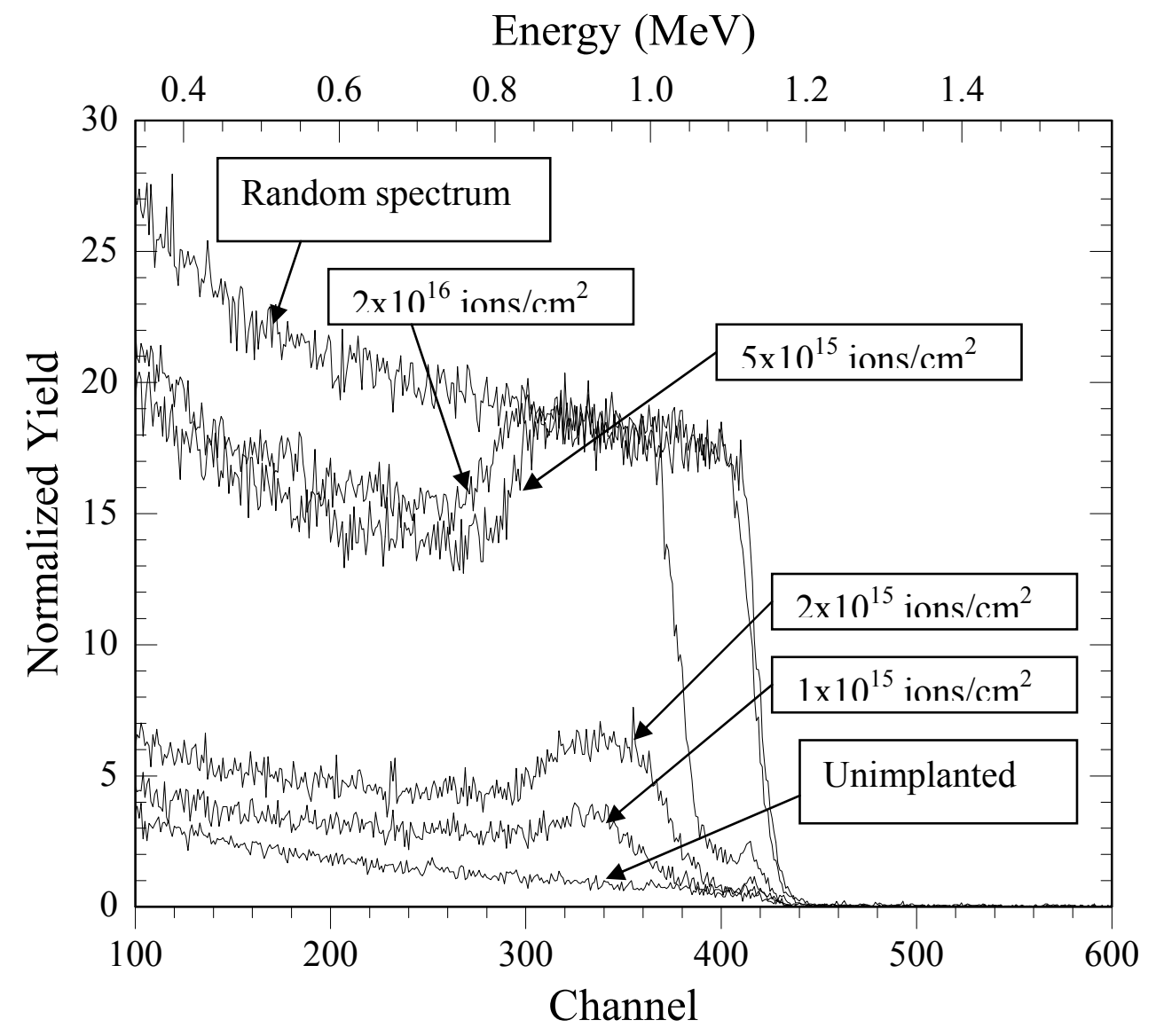

Fig. 2. RBS-channeling spectra of $\mathrm{Si}$ specimens implanted with $300 \mathrm{keV} \mathrm{Ne}$ ions (doses as indicated). 


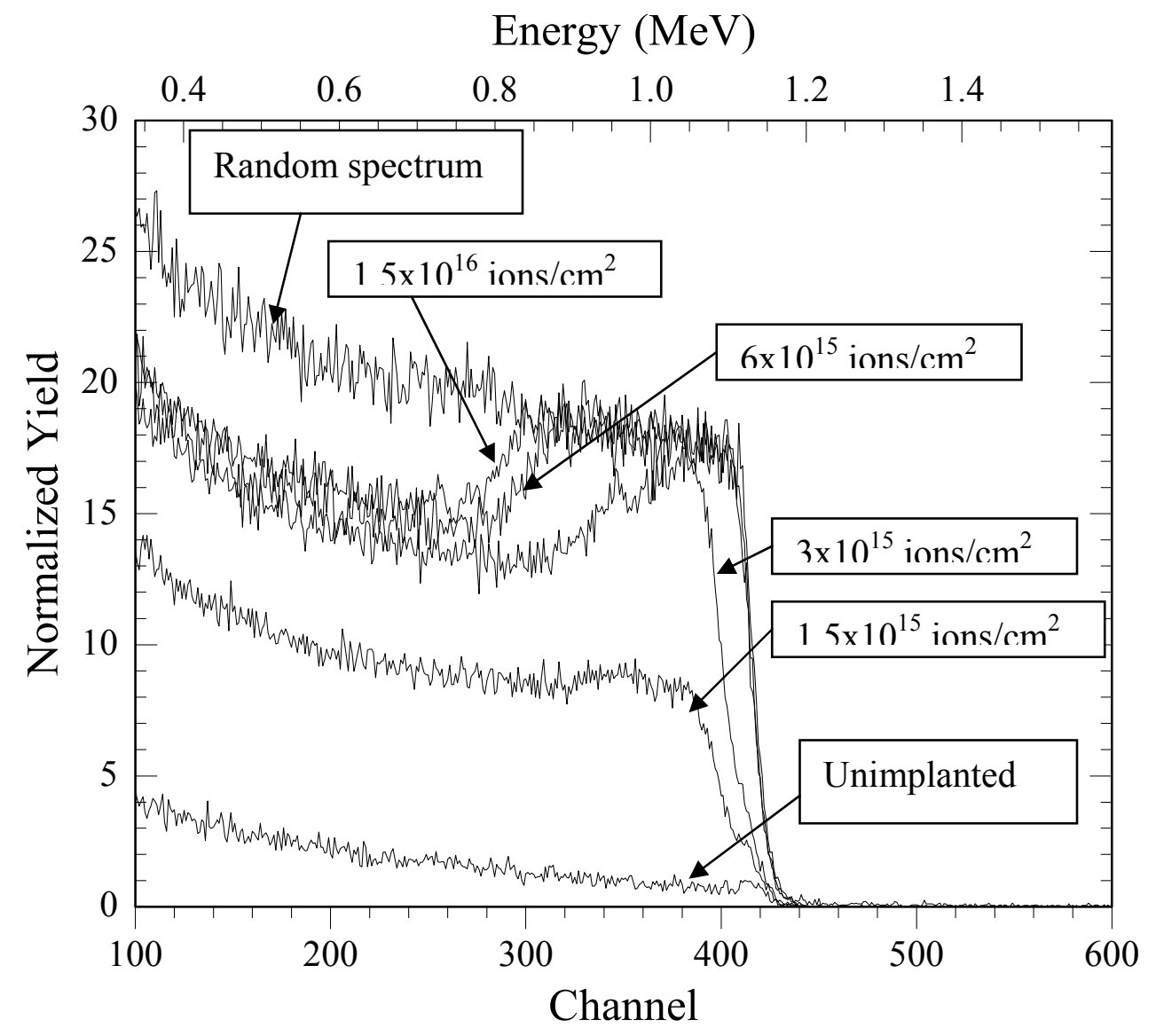

Fig. 3. RBS-channeling spectra of Si specimens implanted with equal doses (indicated) of 100, 200 and $300 \mathrm{keV} \mathrm{Ne}$ ions. 


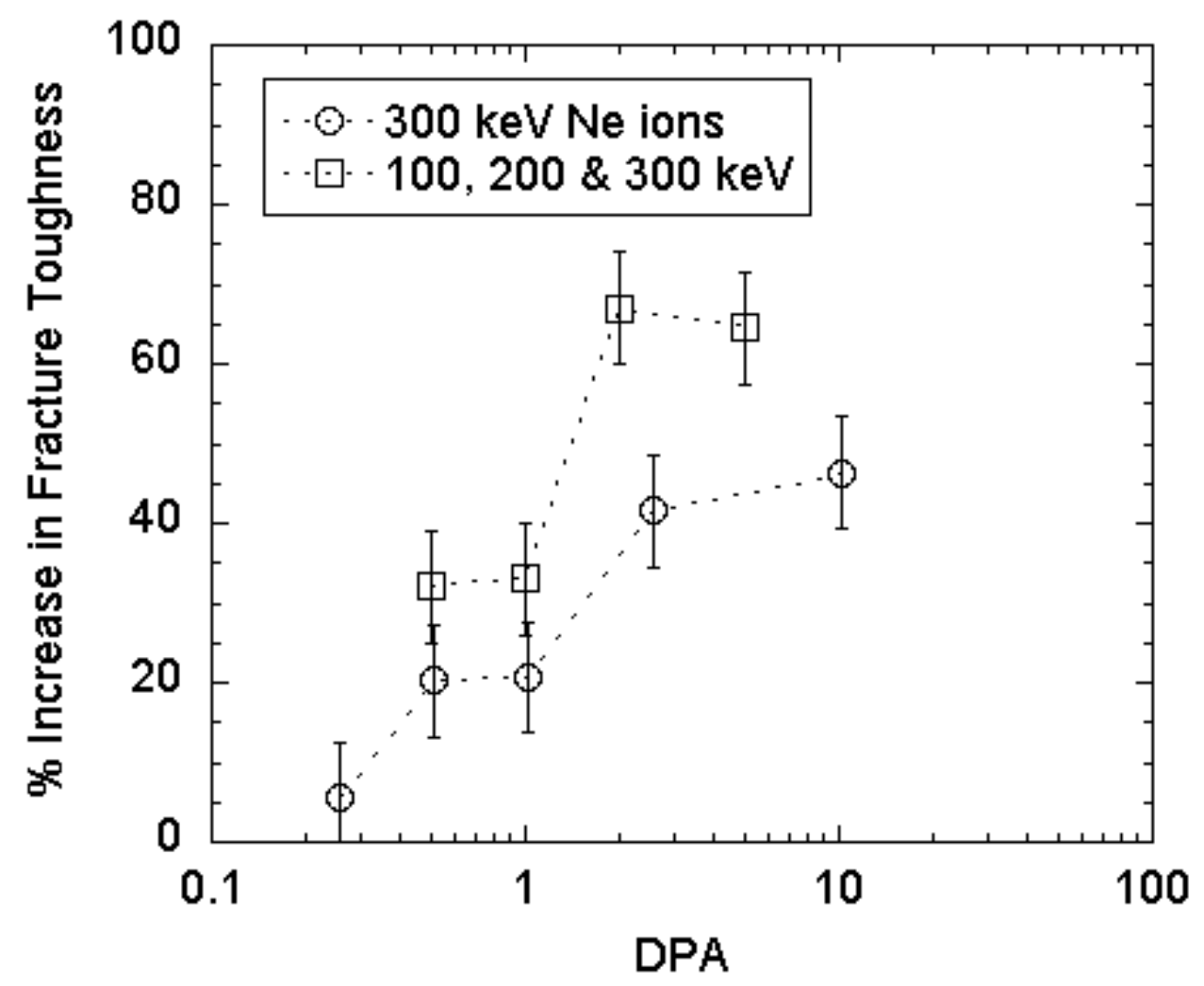

Fig. 4. Increase in fracture toughness (relative to unimplanted specimens) of $\mathrm{Ne}$ implanted $\mathrm{Si}$ specimens: $300 \mathrm{keV}$ ions (circles) and combined 100, 200 and $300 \mathrm{keV}$ ions (squares). 